\title{
Role of N-Linked Glycosylation in PKR2 Trafficking and Signaling
}

\author{
Jissele A. Verdinez ${ }^{1,2,3}$ and Julien A. Sebag 1,2,3* \\ ${ }^{1}$ Department of Molecular Physiology and Biophysics, Carver College of Medicine, University of lowa, lowa City, IA, \\ United States, ${ }^{2}$ Fraternal Order of Eagles Diabetes Research Center, University of lowa, lowa City, IA, United States, ${ }^{3}$ Iowa \\ Neuroscience Institute, University of lowa, lowa City, IA, United States
}

\section{OPEN ACCESS}

Edited by: Hubert Vaudry,

Université de Rouen, France

Reviewed by:

Silvio O. Rizzoli,

Gesellschaft für Wissenschaftliche Datenverarbeitung (MPG), Germany Nils Lambrecht,

VA Long Beach Healthcare System, United States

*Correspondence: Julien A. Sebag Julien-sebag@uiowa.edu

Specialty section: This article was submitted to Neuroendocrine Science, a section of the journal

Frontiers in Neuroscience

Received: 24 June 2021

Accepted: 27 July 2021 Published: 13 August 2021

Citation: Verdinez JA and Sebag JA (2021) Role of N-Linked Glycosylation in PKR2 Trafficking and Signaling.

Front. Neurosci. 15:730417. doi: 10.3389/fnins.2021.730417
Prokineticin receptors are GPCRs involved in several physiological processes including the regulation of energy homeostasis, nociception, and reproductive function. PKRs are inhibited by the endogenous accessory protein MRAP2 which prevents them from trafficking to the plasma membrane. Very little is known about the importance of post-translational modification of PKRs and their role in receptor trafficking and signaling. Here we identify $2 \mathrm{~N}$-linked glycosylation sites within the $\mathrm{N}$-terminal region of PKR2 and demonstrate that glycosylation of PKR2 at position 27 is important for its plasma membrane localization and signaling. Additionally, we show that glycosylation at position 7 results in a decrease in PKR2 signaling through $\mathrm{G} \alpha_{\mathrm{S}}$ without impairing $\mathrm{G} \alpha_{\mathrm{q} / 11}$ signaling.

Keywords: prokineticin receptor, GPCR, MRAP2, trafficking, glycosylation

\section{INTRODUCTION}

Prokineticin receptors (PKRs) are GPCRs expressed both centrally and peripherally (Cheng et al., 2006; Maldonado-Perez et al., 2007; Mohsen et al., 2017). The two members of the family (PKR1 and PKR2) couple both to $\mathrm{G} \alpha_{\mathrm{q} / 11}$ and $\mathrm{G} \alpha_{\mathrm{s}}$ (Chaly et al., 2016; Rouault et al., 2017a). In the periphery, PKRs agonists are known to modulate nociception through regulation of TRPV1 (Hu et al., 2006; Negri et al., 2006; Negri and Lattanzi, 2011; Qiu et al., 2012; Maftei et al., 2020). In the brain, the PKR agonist prokineticin-2 (PK2) acts to decrease food intake in a melanocortin independent manner (Gardiner et al., 2010; Beale et al., 2013; Chaly et al., 2016), however, the mechanism involved is still unclear. Both PKR1 and PKR2 are inhibited by the Melanocortin Receptor Accessory Protein 2 (MRAP2) (Chaly et al., 2016; Rouault et al., 2017a), a single transmembrane protein forming anti-parallel homodimers which has been shown to regulate several GPCRs involved in the control of energy homeostasis (Asai et al., 2013; Sebag et al., 2013; Chaly et al., 2016; Rouault et al., 2017a,b, 2020; Srisai et al., 2017; Bruschetta et al., 2018; Yin et al., 2020; da Fonseca et al., 2021). MRAP2 traps PKRs in intracellular compartments and inhibits their signaling (Chaly et al., 2016; Rouault et al., 2017a). The potent suppression of feeding by PK2 represents a promising novel approach to regulate appetite and possibly a new target for the treatment of obesity, for this reason, it is important to understand how the trafficking and signaling of PKRs are regulated. GPCR trafficking is regulated by numerous factors including homo- and heterodimerization, accessory proteins and post-translational modifications. One such post-translational modification is $\mathrm{N}$-linked glycosylation which has been shown to be important for proper targeting of several GPCRs to the plasma membrane (Duvernay et al., 2005; Patwardhan et al., 2021). While PKR2 possesses two putative N-linked glycosylation sites at positions N7 and N27, their role and importance has not yet been established. In this study, we assessed the role of PKR2 
glycosylation at both putative sites for receptor localization and signaling and the effect of MRAP2 on PKR2 glycosylation.

\section{MATERIALS AND METHODS}

\section{Cell Culture and Transfection}

CHO-K1 were cultured in Dulbecco's modified Eagle's medium (DMEM)/F-12 (Thermo Fisher Scientific) supplemented with 5\% $(\mathrm{v} / \mathrm{v})$ of a 1:1 mix of calf bovine serum and fetal bovine serum and $1 \%$ penicillin-streptomycin. Cells were incubated at $37^{\circ} \mathrm{C}$ in a humidified atmosphere consisting of $5 \% \mathrm{CO}_{2}$. Cells were transfected with LipoD293 DNA transfection reagent (SignaGen Laboratories \#SL100668) following manufacturer's instructions or with PEI transfection reagent (Polysciences Inc.).

\section{Plasmids}

PKR2 plasmid was obtained from the Missouri S\&T cDNA Resource Center. The N-terminal 2HA-tag was added by PCR amplification as previously described (Chaly et al., 2016). The PKR2-GFP plasmid was generated by amplifying PKR2 and GFP sequences separately and assembling them into a pcDNA3.1 + vector using the HiFi DNA assembly kit (NEB). N7Q and N27Q mutations in 2HA-PKR2 and PKR2-GFP were generated by site directed mutagenesis using the Q5-site directed mutagenesis kit (NEB). Cloning and mutations were verified by sequencing. Generation of hMRAP2-3XFlag was previously described (Sebag and Hinkle, 2010). pGloSensor ${ }^{\mathrm{TM}}-22 \mathrm{~F}$ plasmid was obtained from Promega.

\section{Co-immunoprecipitation}

CHO-K1 cells were seeded in 6 well plates were transfected with indicated plasmids using LipoD293 transfection reagent. Cells were lysed in $0.1 \% \mathrm{n}$-dodecyl- $\beta$-maltoside and protease inhibitor cocktail (Sigma catalog no. P8340) in PBS at $4^{\circ} \mathrm{C}$. Lysates were centrifuged $10 \mathrm{~min}$ at $10000 \mathrm{rpm} 4^{\circ} \mathrm{C}$ and supernatants were incubated with either $20 \mu \mathrm{l}$ GFP-nanobody agarose resin or a 1:5000 dilution of M2 Anti-Flag mouse monoclonal antibody overnight at $4{ }^{\circ} \mathrm{C}$. Immune complexes bound to antiFlag antibody were precipitated with Dynabeads Protein G (Life Technologies \#10003D) at $4^{\circ} \mathrm{C}$ for $1 \mathrm{~h}$. Beads were washed 3 times with ice cold lysis buffer. Samples were resuspended in LDS loading buffer with $5 \% \beta$-mercaptoethanol and boiled for $5 \mathrm{~min}$.

\section{Immunoprecipitation}

CHO-K1 cells were seeded in $60 \mathrm{~mm}$ dishes and transfected with the indicated plasmids. Cells were lysed in RIPA buffer supplemented with protease inhibitor cocktail. Lysates were centrifuged and supernatants were incubated with GFPnanobody agarose resin for $1 \mathrm{~h}$ at $4^{\circ} \mathrm{C}$. Nanobody complexes were washed three times with lysis buffer and resuspended in LDS loading buffer containing 5\% $\beta$-mercaptoethanol and boiled for $5 \mathrm{~min}$.

\section{Western Blot}

Proteins were resolved by SDS/PAGE and transferred to PVDF membrane. Membranes were blocked for $1 \mathrm{~h}$ in $5 \%$ non-fat dry milk in PBST, incubated with either mouse anti-flag at 1:5000 or rabbit anti-GFP (Cell Signaling Technologies \#29565) at 1:2000 in blocking buffer o/n $4^{\circ} \mathrm{C}$, washed three times 5 min in PBST, incubated with either anti-mouse or anti-rabbit-HRP secondary antibody in blocking buffer for $1 \mathrm{~h} \mathrm{RT}$, washed three times and imaged using ECL substrate and iBright imager. Densitometry analysis was conducted using photoshop.

\section{Glycosidase Treatments}

$\mathrm{CHO}-\mathrm{K} 1$ cells were seeded in $60 \mathrm{~mm}$ dishes and transfected with PKR2-GFP with or without MRAP2 using LipoD293 transfection reagent. Cells were lysed in RIPA bufer supplemented with protease inhibitor cocktail. Lysates were centrifuged $10 \mathrm{~min}$ at $10000 \mathrm{rpm} 4^{\circ} \mathrm{C}$ and supernatants were incubated with GFP nanobody coated beads for $1 \mathrm{~h}$ at $4^{\circ} \mathrm{C}$. Beads were washed three times in lysis buffer. For PNGase F treatment, samples were resuspended in $10 \mu \mathrm{l}$ glycoprotein denaturing buffer (NEB PNGase F P0708S) and heated at $60^{\circ} \mathrm{C}$ for $10 \mathrm{~min}$. PNGase F reaction mixture was prepared and added to reaction using $2 \mu \mathrm{l}$ of GlycoBuffer 2, $2 \mu \mathrm{l}$ of $10 \%$ NP-40, $1 \mu \mathrm{l}$ PNGase F, and $5 \mu \mathrm{l}$ water. Samples were incubated at $37^{\circ} \mathrm{C}$ for $1 \mathrm{~h}$. Following incubation, $30 \mu \mathrm{l}$ of $2 \times$ LDS loading buffer with $5 \% \beta$-mercaptoethanol was added to each sample. For Endo H treatment, samples were resuspended in $10 \mu \mathrm{l}$ glycoprotein denaturing buffer (NEB Endo $\mathrm{H}$ P0702S) and heated at $60^{\circ} \mathrm{C}$ for $10 \mathrm{~min}$. Endo $\mathrm{H}$ reaction mixture containing $2 \mu \mathrm{l}$ of Glycobuffer $3,1 \mu \mathrm{l}$ Endo $\mathrm{H}$ and $7 \mu \mathrm{l}$ milliq water was added to samples and incubated at $37^{\circ} \mathrm{C}$ for $1 \mathrm{~h}$. Following incubation, $30 \mu \mathrm{l} 2 \times$ LDS loading buffer with $5 \%$ $\beta$-mercaptoethanol was added to each sample.

\section{Fixed Cell ELISA}

CHO-K1 cells were plated in 24 well plates, and triplicate wells were transfected with empty vector, 2HA-PKR2, 2HA PKR2 N7Q, 2HA-PKR2 N27Q, and 2HA-PKR2 N7/27Q. $24 \mathrm{~h}$ following transfection, cells were rinsed with PBS and fixed for $10 \mathrm{~min}$ in $4 \%$ paraformaldehyde (PFA) in PBS. PFA was washed three times with PBS, and cells were blocked with 5\% non-fat dry milk in PBS or RIPA for $30 \mathrm{~min}$ at room temperature on a shaker. Cells were incubated with anti-HA antibody at 1:5000 in blocking buffer for $2 \mathrm{~h}$ at room temperature on a shaker. Cells were washed three times with PBS for $5 \mathrm{~min}$ at room temperature on a shaker and then incubated with anti-mouse HRP at 1:5000 in blocking buffer for $1 \mathrm{~h}$ at room temperature on a shaker. Cells were washed three times for 5 min with PBS. $200 \mu l$ 3,3',5,5'-tetramethylbenzidine (Sigma-Aldrich) was then added until blue color was visible and the reaction was stopped with $200 \mu \mathrm{l} 10 \%$ sulfuric acid. $300 \mu \mathrm{l}$ of each sample was transferred to a 96 well plate, and absorbance was measured at $450 \mathrm{~nm}$ using a Spectramax i3 plate reader (Molecular Devices, Sunnyvale, CA). Each condition was run in triplicate, and experiments were repeated independently at least three times.

\section{Inositol Phosphate Assay}

CHO-K1 cells were seeded in six-well plates and transfected with the indicated plasmids. Cells were then lifted using TrypLE Express (Thermo Fisher Scientific) and resuspended in $400 \mu \mathrm{l}$ DMEM/F12. Cell suspension ( $7 \mu \mathrm{l} /$ well) was transferred to a 
white opaque 384-well plate before adding agonist in stimulation buffer (included in kit). Cells were incubated for $1 \mathrm{~h}$ at $37^{\circ} \mathrm{C}$, lysed and accumulated $\mathrm{IP}_{1}$ was measured using the IP-One kit (Cisbio) following the manufacturer's instructions. Readings were measured with a SpectraMax i3 plate reader (Molecular Devices, Sunnyvale, CA). Each condition was run in triplicate, and experiments were repeated independently at least three times.

\section{cAMP Assay}

$\mathrm{CHO}-\mathrm{K} 1$ cells were seeded in 6 well plates and transfected with indicated plasmids and pGloSensor-22F. Cells were then lifted using TrypLE Express (Thermo Fisher Scientific) and resuspended in $1 \mathrm{ml}$ DMEM/F12. $10 \mu \mathrm{l}$ of cell suspension was transferred to wells of a white clear-bottom 384 well plate and left to adhere overnight. The next day, $10 \mu \mathrm{l}$ of $1.2 \mathrm{mg} / \mathrm{ml} \mathrm{D}$-luciferin (GoldBio, CAS 115144-35) in $\mathrm{CO}_{2}$ Independent Medium was added to each well and allowed to incubate for a minimum of $2 \mathrm{~h}$. A separate 384 well plate with peptide agonist, $\mathrm{PK} 2$, in $\mathrm{CO}_{2}$ Independent Medium at varying concentrations was prepared and placed in the FLIPR Tetra automated kinetic plate reader (Molecular Devices) together with the plate containing the cells. Luminescence was measured in every well at a sampling rate of 4 s. After $2 \mathrm{~min}$ of basal luminescence recording, agonist was injected and luminescence was measured for an additional $13 \mathrm{~min}$. Each condition was run in triplicate, and experiments were repeated independently at least three times.

\section{Immunofluorescence Microscopy}

$\mathrm{CHO}-\mathrm{K} 1$ cells were seeded in six-well plates on glass coverslips and transfected with 2HA-PKR2 or mutant and either empty vector or MRAP2. The next day, coverslips were rinsed with PBS and fixed with 4\% PFA in PBS for $10 \mathrm{~min}$. Coverslips were rinsed three times, permeabilized cells were blocked with 5\% non-fat dry milk in RIPA, non-permeabilized cells with 5\% non-fat dry milk in PBS for $30 \mathrm{~min}$ at room temperature. Cells were then incubated with both mouse anti-HA at 1:5000 and polyclonal rabbit antiMRAP2 at 1:5000 in 5\% non-fat dry milk in PBS overnight at $4^{\circ} \mathrm{C}$. Coverslips were rinsed three times with PBS and incubated with goat-anti rabbit Plus 555 (Invitrogen) and Alexa Fluor 488 goat-anti mouse (Invitrogen) in blocking buffer at 1:5000 dilution. Coverslips were rinsed three times and mounted on glass slides with SlowFade ${ }^{\mathrm{TM}}$ Diamond Antifade Mountant with DAPI (ThermoFisher Scientific \#S36963). Images were obtained with an inverted epifluorescence microscope (Olympus IX83). Experiments were acquired three times independently.

\section{RESULTS}

\section{PKR2 Is Glycosylated at N7 and N27}

Based on the amino acid sequence, PKR2 has $2 \mathrm{~N}$-linked glycosylation sites in the $\mathrm{N}$-terminal region of the receptor at positions 7 and 27 (Figure 1A). To determine if those sites are in fact glycosylated, we transfected CHO cells with PKR2-GFP. Cells were lysed and PKR2 was immunoprecipitated using a GFP nanobody. Samples were then treated with either PNGase F or Endo $\mathrm{H}$ to remove mature or core glycosylation, respectively, before resolving proteins by SDS-PAGE and detecting PKR2 by western blot using anti-GFP antibody. Untreated PKR2 ran as a smear typical of glycosylated GPCRs at around $80-90 \mathrm{kD}$ (Figure 1B). PNGase F treatment resulted in increased mobility of PKR2 consistent with deglycosylation (Figure 1B). The high molecular weight smear was, however, not affected by Endo $\mathrm{H}$ treatment, thus identifying this band as the mature glycosylated form of the receptor (Figure 1B). We had previously shown that PKR2 trafficking to the plasma membrane and signaling is inhibited by the accessory protein MRAP2. To test whether the interaction of MRAP2 with PKR2 results in a decrease in receptor glycosylation, cells were transfected with PKR2GFP and MRAP2-3XFlag. Samples were incubated with either anti-GFP nanobody coated beads or with anti-Flag antibody to immunoprecipitate PKR2 or MRAP2, respectively. Samples were resolved by SDS-PAGE and proteins were detected by western blot using either anti-GFP or anti-Flag antibodies. In samples immunoprecipitated with the GFP nanobodies, the receptor separated in two bands (glycosylated and immature receptor), however, in samples immunoprecipitated with anti-flag antibody, the immature form of the receptor was enriched (Figure 1C), thus suggesting that interaction of MRAP2 preferentially interacts with the non-glycosylated form of PKR2 and possibly prevents its glycosylation. Co-immunoprecipitation of PKR2 and MRAP2 was also confirmed by detection of MRAP2 in samples immunoprecipitated with anti-GFP nanobodies (Figure 1D). To determine if both N7 and N27 can be glycosylated, we generated PRK2 constructs with substitution of N7 and/or N27 with a glutamine (N7Q, N27Q, and N7/27Q mutants). Mutants were transfected in CHO cells with or without MRAP2. Unmodified PKR2-GFP ran as two bands as described earlier (Figure 1E). The higher band representing the mature receptor and the lower band the core glycosylated receptor. Substitution of either N7 or N27 to Q resulted in increased mobility of both bands thus demonstrating that both sites can be glycosylated (Figure 1E). Substitution of both N7 and N27 to Q resulted in the complete loss of mature and core glycosylated receptor and only the immature non-glycosylated receptor was detected (Figure 1E). In the presence of MRAP2, the same profile is observed, however, in every case the relative amount of mature receptor compared to the immature form is greatly decreased (Figure 1E). Densitometric analysis shows a significant decreased ratio of glycosylated over non-glycosylated PKR2 in the presence of MRAP2 for WT PKR2 (Figure 1F), N7Q mutant (Figure 1G), and N27Q mutant (Figure 1H), thus further demonstrating that the presence of MRAP2 prevents PKR2 glycosylation at both N7 and N27 sites.

\section{Glycosylation at N27 Is Essential for PKR2 Trafficking}

To assess the importance of glycosylation at N7 and N27 of PKR2 for receptor trafficking, PKR2, PKR2-N7Q, PKR2-N27Q, and PKR2-N7/27Q N-terminally fused to 2-HA tags were transfected in $\mathrm{CHO}$ cells. Localization of WT and mutant receptors was then determined by immunofluorescence microscopy in nonpermeabilized and permeabilized cells using anti-HA antibody. 


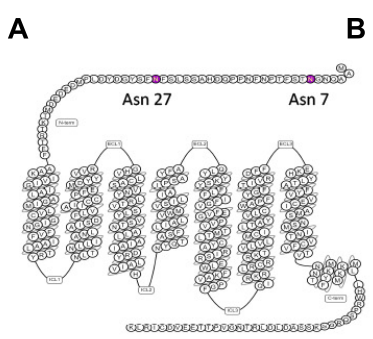

E

PKR2 GFP EV WT N7Q N27Q NQ2 WT N7Q N27Q NQ2 MRAP2-3F - $\quad-\quad-\quad-\quad+\quad+\quad+\quad++$

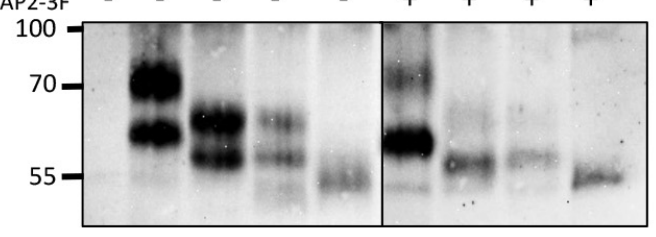

F
C

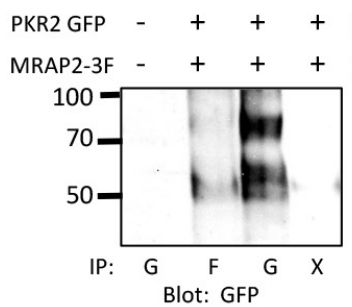

D

G
H

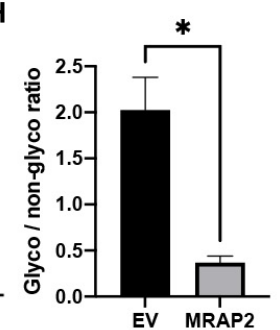

I
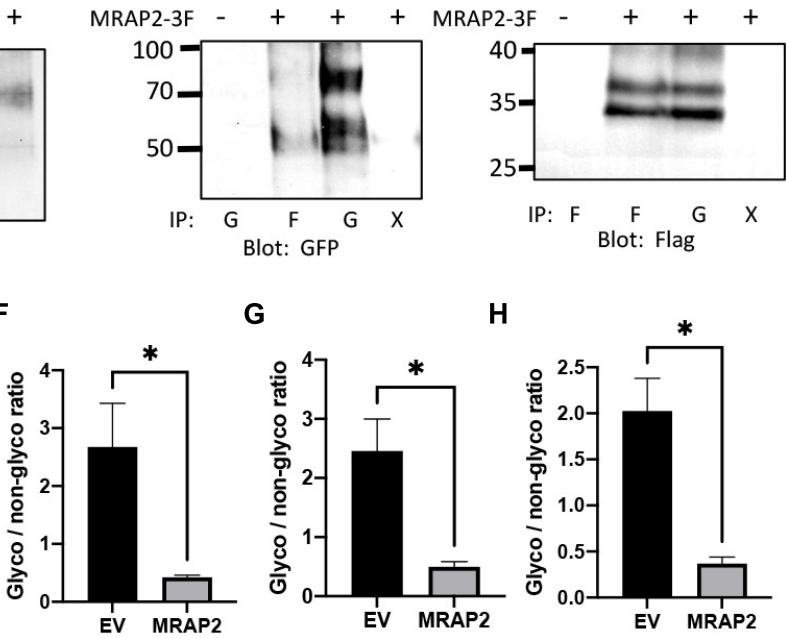
PKR2

PKR2-N7Q
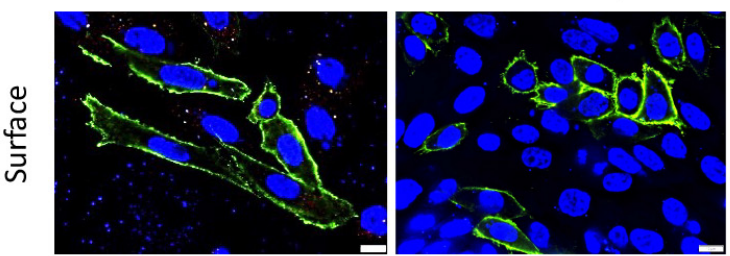

PKR2-N27Q

PKR2-N7-27Q
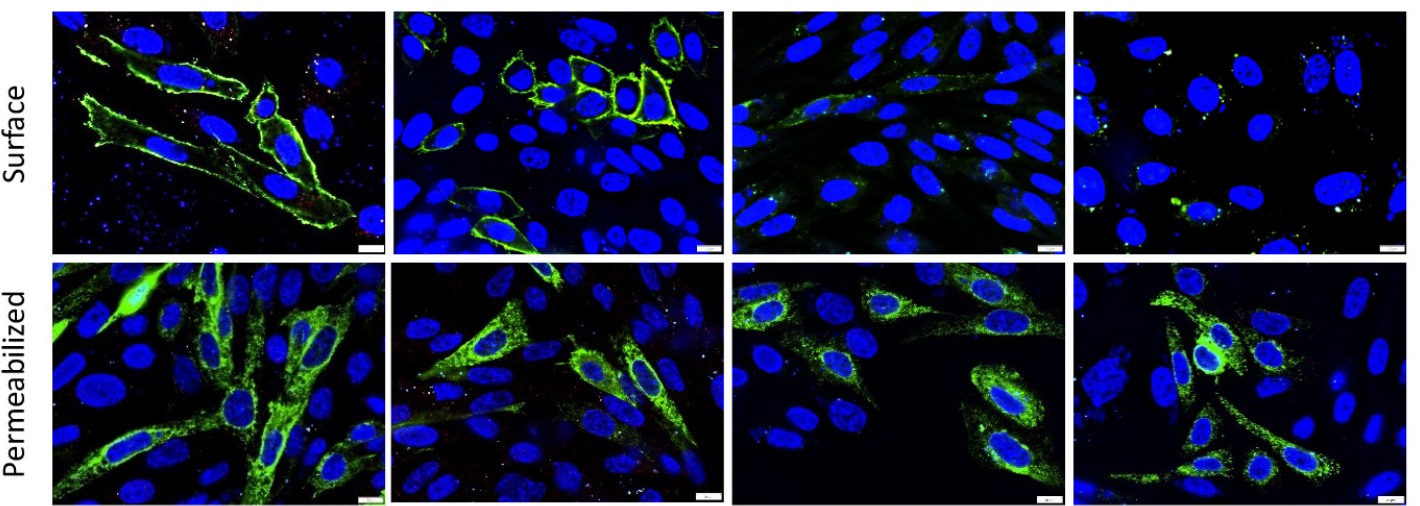

$\mathbf{J}$
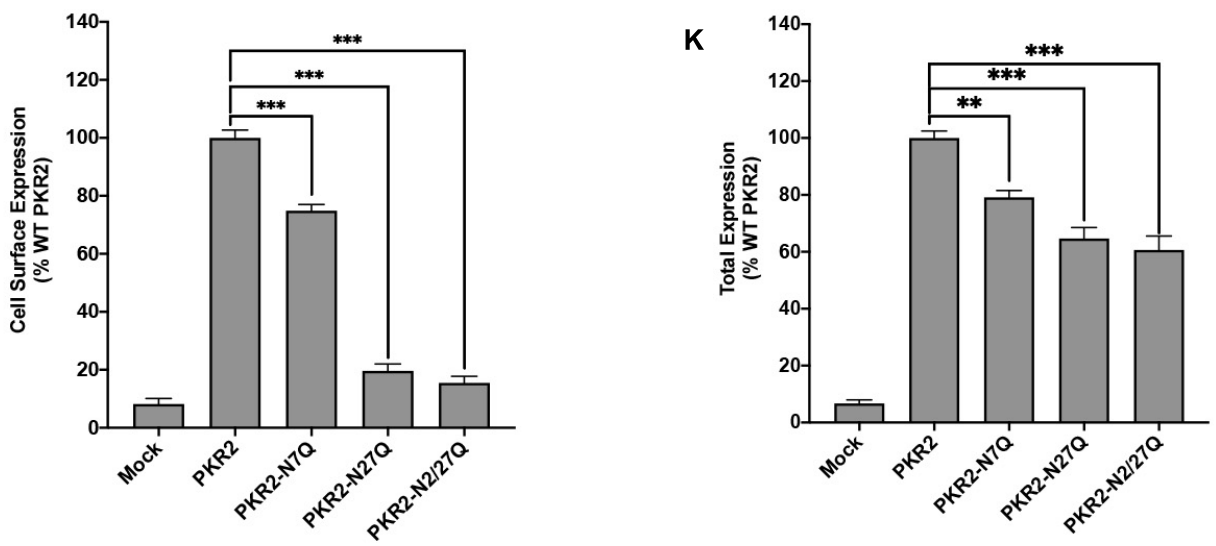

FIGURE 1 | Role of N-linked glycosylation in PKR2 trafficking. (A) Schematic depiction of human PKR2 glycosylation sites. (B) Western blot detection of untreated PKR2-GFP or PKR2-GFP treated with PNGase F or EndoH. (C,D) Western blot detection of PKR2 (C) or MRAP2 (D) following co-immunoprecipitation of PKR2-GFP and MRAP2-3XFLAG from transfected CHO cells. Immunoprecipitation was performed using beads coated with GFP nanobodies "G" or anti-Flag antibody "F." "X" indicates that no antibody was used for the IP (beads only). (E) Western blot detection of PKR2-GFP alone or with MRAP2 treated or not with PNGase F or Endo H. (F-H) Densitometry analysis measuring the ration of glycosylated to non-glycosylated PKR2 in the presence and absence of MRAP2 for WT (F), N7Q mutant (G), and N27Q mutant (H). (I) Immunofluorescence detection of 2HA-PKR2, 2HA-PKR2-N7Q, 2HA-PKR2-N27Q, and 2HA-PKR2-N7/27 in non-permeabilized and permeabilized cells. (J,K) Cell ELISA detection of 2HA-tagged PKR2 and PKR2 mutants in non-permeabilized (J) and permeabilized (K) cells. Errors are mean \pm SEM. ${ }^{*} p<0.05,{ }^{* *} p<0.01,{ }^{* * *} p<0.001$ 


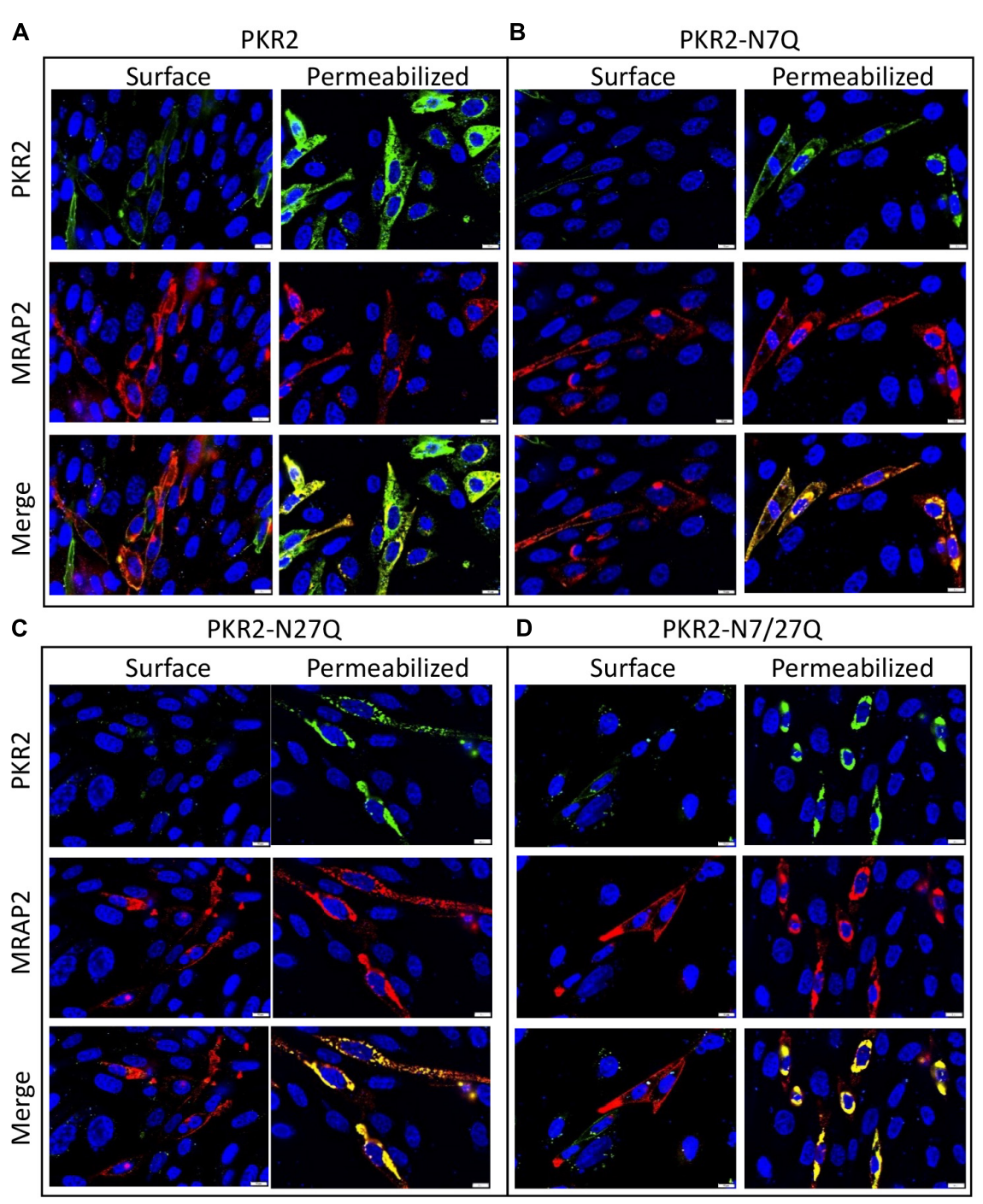

FIGURE 2 | Localization of PKR2 and glycosylation mutants PKR2 expressed with MRAP2. (A-D) Immunofluorescence detection of MRAP2 and 2HA-PK2 (A), 2HA-PKR2-N7Q (B), 2HA-PKR2-N27Q (C), and 2HA-PKR2-N7/27Q (D) in non-permeabilized or permeabilized cells. PKR2 is shown in green, MRAP2 is shown in red and yellow depicts co-localization. Nuclei are shown in blue (DAPI).

Whereas PKR2 and PKR2-N7Q were readily detectable at the surface of non-permeabilized cells, PKR2-N27Q and PKR2N7/27Q were not (Figure 1I). WT and all mutants were detected in permeabilized cells, thus confirming expression (Figure 1I). Results from this experiment suggest that glycosylation of N27 is essential for PKR2 trafficking to the plasma membrane. To quantitatively verify this finding, PKR2 and mutated PKR2 were transfected in $\mathrm{CHO}$ cells before measuring surface and total expression of receptor in non-permeabilized and permeabilized cells, respectively, by in-cell ELISA using anti-HA antibody. In agreement with the microscopy results, the N7Q mutant was readily detectable at the cell surface, albeit at a lower density compared to the non-mutated receptor (Figure 1J). In contrast, the surface density of N27Q and N7/27Q mutants were drastically reduced ( $\sim 90 \%$ decrease) compared to control receptor (Figure 1J). The total expression of mutated PKR2 measured in permeabilized cells was only reduced by $20-40 \%$ (Figure 1K), thus, the decrease in expression is not sufficient to explain the drastic loss of surface expression for PKR2-N27Q and N7/27Q. These results suggest that glycosylation at N27 is essential for receptor trafficking to the cell surface and may play a role in receptor stability.

\section{MRAP2 Prevents PKR2 From Trafficking to the Plasma Membrane Regardless of Glycosylation State}

We had previously shown that surface density of PKR2 was reduced in the presence of MRAP2. Here we tested if receptor glycosylation alters the effect of MRAP2 on PKR2 localization. To this end, we performed immunofluorescence microscopy in CHO cells transfected with PKR2, PKR2-N7Q, 


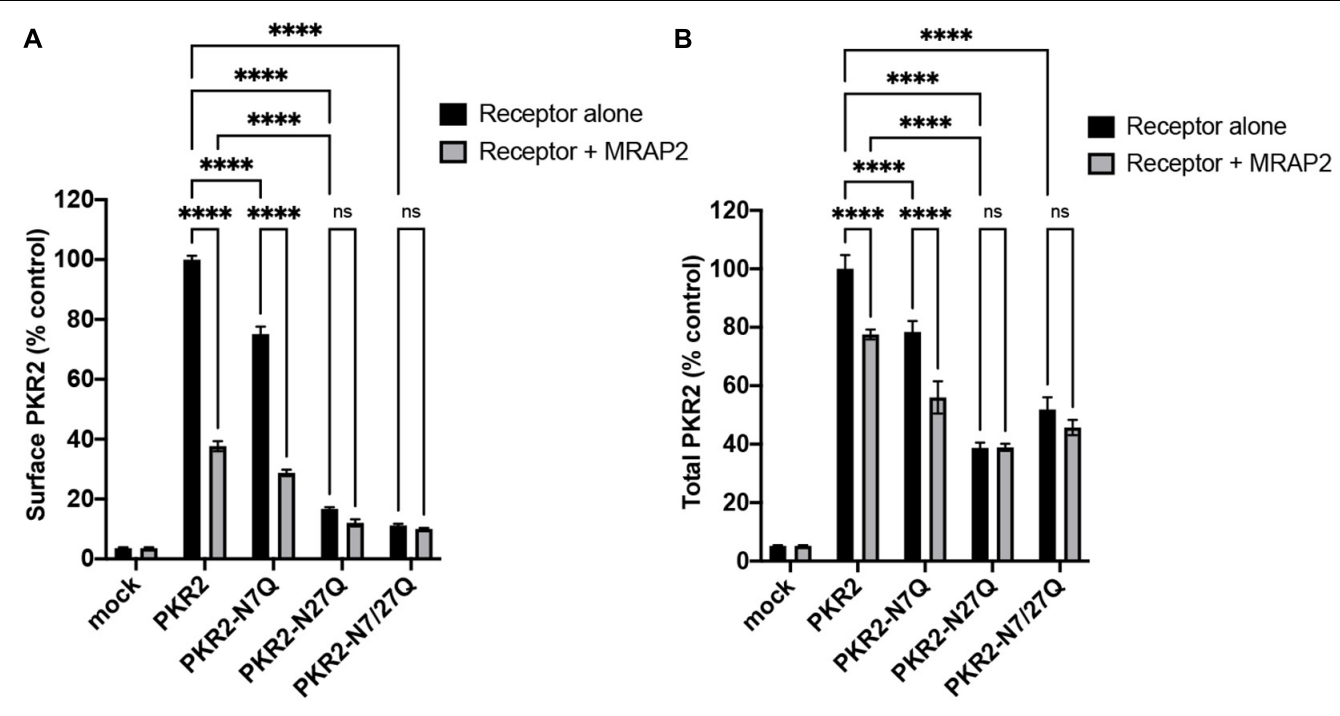

FIGURE 3 | Surface and total quantitation of PKR2 and PKR2 mutants with and without MRAP2. (A) Cell ELISA detection of 2HA-PKR2 and 2HA-PKR2 mutants expressed alone or with MRAP2 in non-permeabilized cells. (B) Cell ELISA detection of 2HA-PKR2 and 2HA-PKR2 mutants expressed alone or with MRAP2 in permeabilized cells. Errors are mean \pm SEM. ${ }^{* * * *} p<0.0001$.

PKR2-N27Q, or PKR2-N7/27Q in the presence of MRAP2. Whereas in the absence of MRAP2, PKR2, and PKR2-N7Q were detectable on the surface of non-permeabilized cells (Figure 1F), detection of those proteins was drastically reduced by MRAP2 expression (Figures 2A,B). For PKR2-N27Q and PKR2-N7/27Q, no receptor was detected at the plasma membrane regardless of MRAP2 expression (Figures 1F, 2C,D). Images of permeabilized cells show that MRAP2 causes a retention of PKR2 and PKR2N7Q in intracellular compartments (Figures 2A,B). Additionally, MRAP2 staining displayed extensive co-localization with PKR2 and PKR2 mutants (Figures 2A-D).

To quantitatively measure the effect of MRAP2 on the trafficking of PKR2 and glycosylation mutants of PKR2, 2HAPKR2, 2HA-PKR2-N7Q, 2HA-PKR2-N27Q, and 2HA-PKR2N7/27Q were transfected alone or with MRAP2 before measuring surface density and total expression by cell ELISA. Both WT and PKR2-N7Q were highly expressed at the cell surface and surface density was drastically reduced by MRAP2 for both (Figure 3A). Surface expression of PKR2-N27Q and PKR2-N7/27Q was very low regardless of MRAP2 expression (Figure 3A). Total expression of all receptor forms was readily detectable, however, expression of PKR2-N27Q and PKR2-N7/27Q were about half of the unmodified receptor (Figure 3B). MRAP2 expression caused a small decrease in total expression of PKR2 and PKR2N7Q but did not alter the total expression of PKR2-N27Q and N-7/27Q (Figure 3B).

\section{Glycosylation Is Important for PKR2 Signaling}

Prokineticin receptors can signal through both $\mathrm{G} \alpha_{\mathrm{q} / 11}$ and $\mathrm{G} \alpha_{\mathrm{s}}$. To determine the importance of glycosylation for PKR2 signaling through $\mathrm{G} \alpha_{\mathrm{q} / 11}$, we measured PK2-mediated IP3 production in $\mathrm{CHO}$ cells expressing either PKR2, PKR2-N7Q, PKR2-N27Q, or PKR2-N7/27Q in the presence or absence of MRAP2. PK2 caused a concentration dependent increase in IP3 production in cells expressing PKR2 (Figure 4A). As previously shown, MRAP2 drastically inhibited PKR2 signaling (Figure 4A). Remarkably, mutation of asparagine 7 did not alter PKR2 signaling through $\mathrm{G} \alpha_{\mathrm{q} / 11}$ or the inhibition caused by MRAP2 (Figure 4B). In contrast, mutation of N27 (Figure 4C) or both N7 and N27 (Figure 4D) resulted in a significant decrease in PK2 efficacy and IP3 production, thus demonstrating that glycosylation at N27 is important for PKR2 function. The decrease in PK2 efficacy is likely due to the decrease in surface receptor density when N27 is not glycosylated. We then tested the effect of removing the N7 and/or N27 glycosylation sites on PKR2 signaling through $\mathrm{G} \alpha_{\mathrm{s}}$ by measuring PK2-stimulated cAMP production. Here again, PK2 caused a concentration-dependent increase in CAMP production in cells expressing PKR2 and cAMP production was drastically inhibited by MRAP2 (Figure 4E). Interestingly, whereas the N7Q mutation had no adverse effect on PKR2-mediated IP3 production (Figure 4B), loss of glycosylation at N7 resulted in a significant decrease in PK2-stimulated cAMP production (Figure 4F), thus suggesting that glycosylation at N7 is not essential for signaling through $\mathrm{G} \alpha_{\mathrm{q} / 11}$ but is important for $\mathrm{G} \alpha_{\mathrm{s}}$ activation. MRAP2 further inhibited signaling of PKR2-N7Q. $\mathrm{G} \alpha_{\mathrm{s}}$ signaling of both $\mathrm{N} 27 \mathrm{Q}$ and N7/27Q mutants were greatly reduced (Figures $\mathbf{4 G}, \mathbf{H}$ ), in agreement with the large decrease in surface density of those mutants.

\section{DISCUSSION}

Prokineticin receptors play numerous important physiological roles like regulating feeding, pain or reproduction. Deletion of PKR2 in rodents results in multiple severe developmental 
A

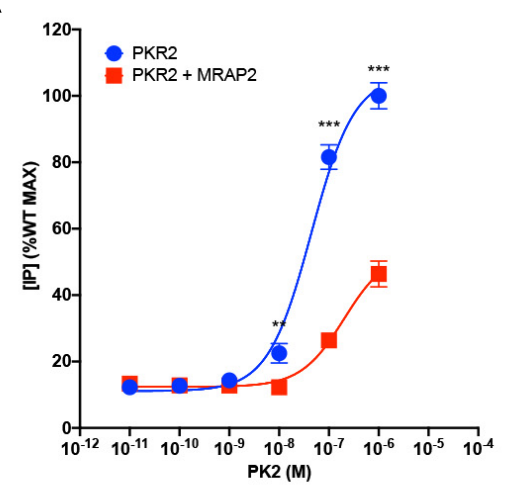

C

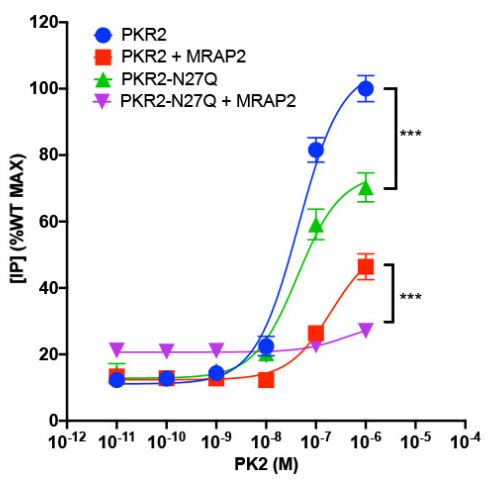

E

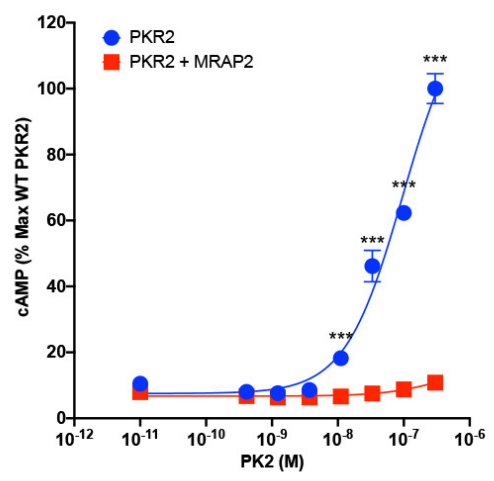

G

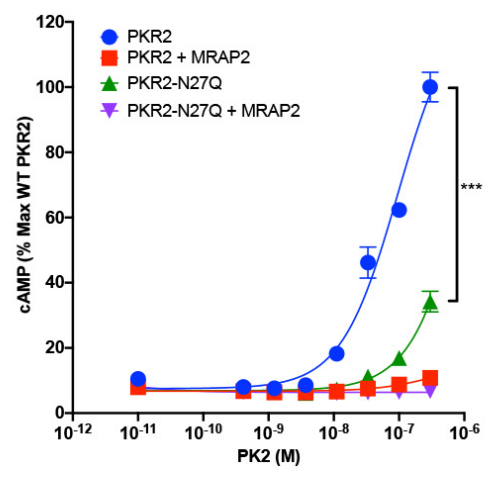

B

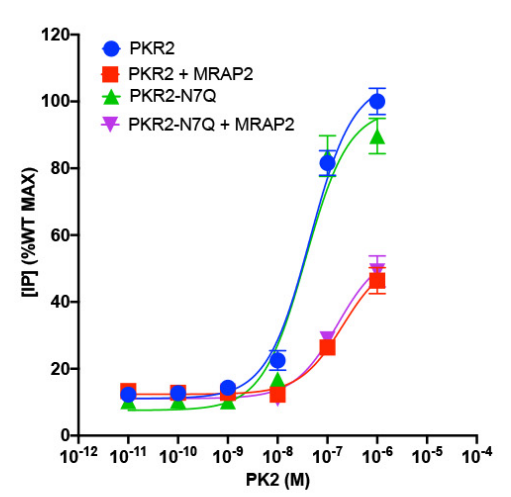

D

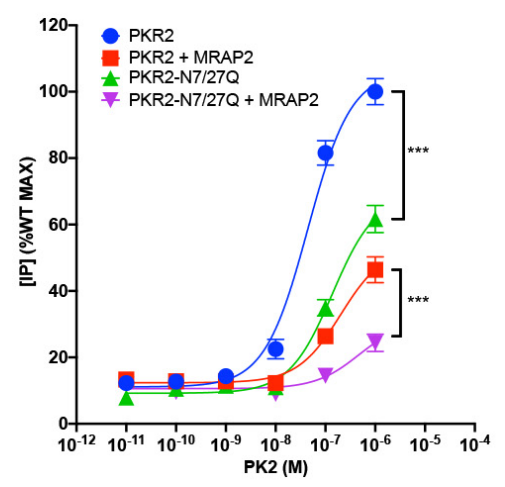

$\mathbf{F}$

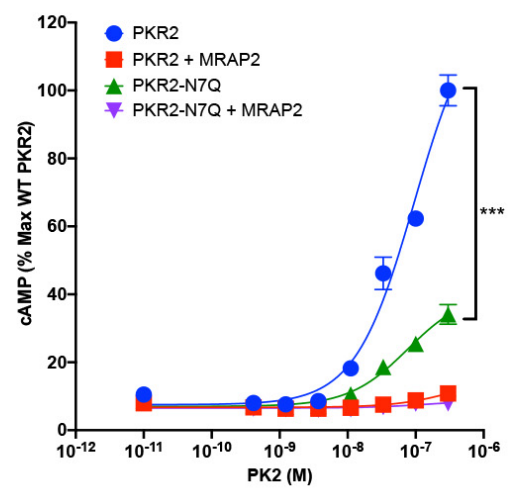

H

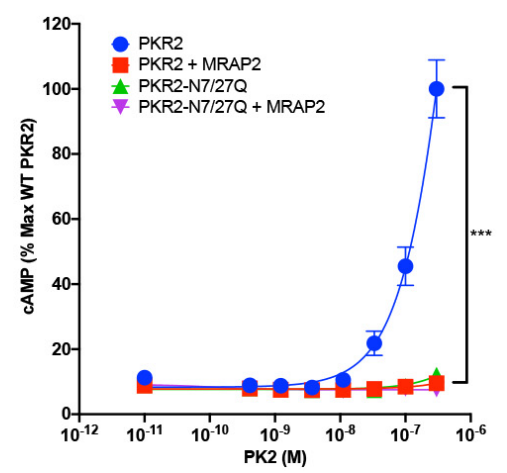

FIGURE 4 | Role of $\mathrm{N}$-glycosylation in PKR2 signaling. (A-D) Inositol phosphate detection in cells expressing PKR2 (A), PKR2-N7Q (B), PKR2-N27Q (C), or PKR2-N7/27Q (D) with or without MRAP2 and stimulated with the indicated concentration of PK2. (E-H) CAMP measurement in cells expressing PKR2 (E), PKR2-N7Q (F), PKR2-N27Q (G), or PKR2-N7/27Q (H) with or without MRAP2 and stimulated with the indicated concentration of PK2. Errors are mean \pm SEM. ${ }^{* *} p<0.01,{ }^{* \star *} p<0.001$. 
phenotypes including underdeveloped olfactory bulbs, sterility, altered thermogenesis, and circadian rhythm reminiscent of Kallmann syndrome (Matsumoto et al., 2006). Like most GPCRs, PKR2 can be glycosylated at the N-terminus, however, the importance of this post-translational modification for PKR2 trafficking and signaling was not established. In many cases, $\mathrm{N}$-linked glycosylation of GPCRs was shown to be important for receptor folding and trafficking to the plasma membrane (Karpa et al., 1999; Lanctot et al., 1999; Compton et al., 2002; Michineau et al., 2004; Wang et al., 2020; Patwardhan et al., 2021) whereas in others glycosylation appears non-essential (van Koppen and Nathanson, 1990; Fukushima et al., 1995; Karpa et al., 1999; Kozielewicz et al., 2017; Patwardhan et al., 2021). We identified two glycosylation sites on the N-terminal region of PKR2 and found that while glycosylation at position 27 is essential for PKR2 trafficking to the plasma membrane, glycosylation at position 7 is not. We had previously identified MRAP2 as an interacting protein of PKR2 and showed that MRAP2 sequesters PKR2 in intracellular compartments (Chaly et al., 2016; Rouault et al., 2017b). Here we show that in the presence of MRAP2, PKR2 glycosylation is largely prevented, which is consistent with the receptor being retained in the endoplasmic reticulum (ER). The lack of glycosylation of PKR2 in the presence of MRAP2 suggests that PKR2 is retained in the ER rather than trafficked to the membrane and rapidly internalized. MRAP2 prevented trafficking of both the unmodified and N7Q PKR2 mutants, thus suggesting that N7 is not important for MRAP2-mediated inhibition of PKR2. Mutation of N27 resulted in a decrease in both $\mathrm{G} \alpha_{\mathrm{s}}$ and $\mathrm{G} \alpha_{\mathrm{q} / 11}$ signaling, consistent with the decrease in surface receptor density. Surprisingly, however, mutation of N7

\section{REFERENCES}

Asai, M., Ramachandrappa, S., Joachim, M., Shen, Y., Zhang, R., Nuthalapati, N., et al. (2013). Loss of function of the melanocortin 2 receptor accessory protein 2 is associated with mammalian obesity. Science 341, 275-278. doi: $10.1126 /$ science. 1233000

Beale, K., Gardiner, J. V., Bewick, G. A., Hostomska, K., Patel, N. A., Hussain, S. S., et al. (2013). Peripheral administration of prokineticin 2 potently reduces food intake and body weight in mice via the brainstem. Br. J. Pharmacol. 168, 403-410. doi: 10.1111/j.1476-5381.2012.02191.x

Bruschetta, G., Kim, J. D., Diano, S., and Chan, L. F. (2018). Overexpression of melanocortin 2 receptor accessory protein 2 (MRAP2) in adult paraventricular MC4R neurons regulates energy intake and expenditure. Mol. Metab. 18, 79-87. doi: 10.1016/j.molmet.2018.09.010

Chaly, A. L., Srisai, D., Gardner, E. E., and Sebag, J. A. (2016). The Melanocortin Receptor Accessory Protein 2 promotes food intake through inhibition of the Prokineticin Receptor-1. eLife 5:e12397.

Cheng, M. Y., Leslie, F. M., and Zhou, Q. Y. (2006). Expression of prokineticins and their receptors in the adult mouse brain. J. Comp. Neurol. 498, 796-809. doi: 10.1002/cne.21087

Compton, S. J., Sandhu, S., Wijesuriya, S. J., and Hollenberg, M. D. (2002). Glycosylation of human proteinase-activated receptor-2 (hPAR2): role in cell surface expression and signalling. Biochem. J. 368, 495-505. doi: 10.1042/ bj20020706

da Fonseca, A. C. P., Abreu, G. M., Zembrzuski, V. M., Campos Junior, M., Carneiro, J. R. I., Nogueira Neto, J. F., et al. (2021). Study of LEP, MRAP2 and POMC genes as potential causes of severe obesity in Brazilian patients. Eat. Weight. Disord. 26, 1399-1408. doi: 10.1007/s40519-020-00946-z resulted in normal $\mathrm{G} \alpha_{\mathrm{q} / 11}$ signaling but drastically reduced $\mathrm{G} \alpha_{\mathrm{s}}$ coupling even though surface receptor density was only slightly reduced. This is particularly interesting because, whereas the importance of N-linked glycosylation for receptor trafficking has been reported for numerous GPCR, signaling bias as a result of a change in receptor glycosylation has only been identified for a few receptors (Marada et al., 2015; Soto et al., 2015; Patwardhan et al., 2021). Futures studies will need to be conducted to determine how glycosylation at position 7 modifies G-protein selectivity for PKR2 and the mechanism through which MRAP2 prevents PKR2 from exiting the ER.

\section{DATA AVAILABILITY STATEMENT}

The raw data supporting the conclusions of this article will be made available by the authors, without undue reservation.

\section{AUTHOR CONTRIBUTIONS}

JAV conducted experiments and wrote the manuscript. JAS supervised the projects and edited the manuscript. Both authors analyzed the data, contributed to the article, and approved the submitted version.

\section{FUNDING}

This work was supported by the NIH grant RO3TR002838.

Duvernay, M. T., Filipeanu, C. M., and Wu, G. (2005). The regulatory mechanisms of export trafficking of G protein-coupled receptors. Cell. Signal. 17, 1457-1465. doi: 10.1016/j.cellsig.2005.05.020

Fukushima, Y., Oka, Y., Saitoh, T., Katagiri, H., Asano, T., Matsuhashi, N., et al. (1995). Structural and functional analysis of the canine histamine H2 receptor by site-directed mutagenesis: $\mathrm{N}$-glycosylation is not vital for its action. Biochem. J. 310(Pt 2), 553-558. doi: 10.1042/bj3100553

Gardiner, J. V., Bataveljic, A., Patel, N. A., Bewick, G. A., Roy, D., Campbell, D., et al. (2010). Prokineticin 2 is a hypothalamic neuropeptide that potently inhibits food intake. Diabetes Metab. Res. Rev. 59, 397-406. doi: 10.2337/db091198

Hu, W. P., Zhang, C., Li, J. D., Luo, Z. D., Amadesi, S., Bunnett, N., et al. (2006). Impaired pain sensation in mice lacking prokineticin 2. Mol. Pain 2:35.

Karpa, K. D., Lidow, M. S., Pickering, M. T., Levenson, R., and Bergson, C. (1999). $\mathrm{N}$-linked glycosylation is required for plasma membrane localization of D5, but not D1, dopamine receptors in transfected mammalian cells. Mol. Pharmacol. 56, 1071-1078. doi: 10.1124/mol.56.5.1071

Kozielewicz, P., Alomar, H., Yusof, S., Grafton, G., Cooper, A. J., Curnow, S. J., et al. (2017). N-glycosylation and expression in human tissues of the orphan GPR61 receptor. FEBS Open Biol. 7, 1982-1993. doi: 10.1002/2211-5463.12339

Lanctot, P. M., Leclerc, P. C., Escher, E., Leduc, R., and Guillemette, G. (1999). Role of $\mathrm{N}$-glycosylation in the expression and functional properties of human AT1 receptor. Biochemistry 38, 8621-8627. doi: 10.1021/bi9830516

Maftei, D., Vellani, V., Artico, M., Giacomoni, C., Severini, C., and Lattanzi, R. (2020). Abnormal pain sensation in mice lacking the prokineticin receptor PKR2: interaction of PKR2 with Transient Receptor Potential TRPV1 and TRPA1. Neuroscience 427, 16-28. doi: 10.1016/j.neuroscience.2019. 12.003 
Maldonado-Perez, D., Evans, J., Denison, F., Millar, R. P., and Jabbour, H. N. (2007). Potential roles of the prokineticins in reproduction. Trends Endocrinol. Metab. 18, 66-72. doi: 10.1016/j.tem.2006.12.002

Marada, S., Navarro, G., Truong, A., Stewart, D. P., Arensdorf, A. M., Nachtergaele, S., et al. (2015). Functional divergence in the role of N-linked glycosylation in smoothened signaling. PLoS Genet. 11:e1005473. doi: 10.1371/journal.pgen. 1005473

Matsumoto, S., Yamazaki, C., Masumoto, K. H., Nagano, M., Naito, M., Soga, T., et al. (2006). Abnormal development of the olfactory bulb and reproductive system in mice lacking prokineticin receptor PKR2. Proc. Natl. Acad. Sci. U.S.A. 103, 4140-4145. doi: 10.1073/pnas.0508881103

Michineau, S., Muller, L., Pizard, A., Alhenc-Gelas, F., and Rajerison, R. M. (2004). $\mathrm{N}$-linked glycosylation of the human bradykinin B2 receptor is required for optimal cell-surface expression and coupling. Biol. Chem. 385, 49-57.

Mohsen, Z., Sim, H., Garcia-Galiano, D., Han, X., Bellefontaine, N., Saunders, T. L., et al. (2017). Sexually dimorphic distribution of Prokr2 neurons revealed by the Prokr2-Cre mouse model. Brain Struct. Funct. 222, 4111-4129. doi: 10.1007/s00429-017-1456-5

Negri, L., and Lattanzi, R. (2011). Bv8-prokineticins and their receptors: modulators of pain. Curr. Pharm. Biotechnol. 12, 1720-1727. doi: 10.2174/ 138920111798357410

Negri, L., Lattanzi, R., Giannini, E., Colucci, M., Margheriti, F., Melchiorri, P., et al. (2006). Impaired nociception and inflammatory pain sensation in mice lacking the prokineticin receptor PKR1: focus on interaction between PKR1 and the capsaicin receptor TRPV1 in pain behavior. J. Neurosci. 26, 6716-6727. doi: 10.1523/jneurosci.5403-05.2006

Patwardhan, A., Cheng, N., and Trejo, J. (2021). Post-translational modifications of $\mathrm{G}$ protein-coupled receptors control cellular signaling dynamics in space and time. Pharmacol. Rev. 73, 120-151. doi: 10.1124/pharmrev.120.000082

Qiu, C. Y., Liu, Y. Q., Qiu, F., Wu, J., Zhou, Q. Y., and Hu, W. P. (2012). Prokineticin 2 potentiates acid-sensing ion channel activity in rat dorsal root ganglion neurons. J. Neuroinflammation 9:108.

Rouault, A. A. J., Lee, A. A., and Sebag, J. A. (2017a). Regions of MRAP2 required for the inhibition of orexin and prokineticin receptor signaling. Biochim. Biophys. Acta Mol. Cell Res. 1864, 2322-2329. doi: 10.1016/j.bbamcr.2017.09. 008

Rouault, A. A. J., Srinivasan, D. K., Yin, T. C., Lee, A. A., and Sebag, J. A. (2017b). Melanocortin receptor accessory proteins (MRAPs): functions in the melanocortin system and beyond. Biochim. Biophys. Acta Mol. Basis Dis. 1863, 2462-2467. doi: 10.1016/j.bbadis.2017.05.008

Rouault, A. A. J., Rosselli-Murai, L. K., Hernandez, C. C., Gimenez, L. E., Tall, G. G., and Sebag, J. A. (2020). The GPCR accessory protein MRAP2 regulates both biased signaling and constitutive activity of the ghrelin receptor GHSR1a. Sci. Signal. 13:eaax4569. doi: 10.1126/scisignal.aax4569

Sebag, J. A., and Hinkle, P. M. (2010). Regulation of G protein-coupled receptor signaling: specific dominant-negative effects of melanocortin 2 receptor accessory protein 2. Sci. Signal. 3:ra28. doi: 10.1126/scisignal.2000593

Sebag, J. A., Zhang, C., Hinkle, P. M., Bradshaw, A. M., and Cone, R. D. (2013). Developmental control of the melanocortin-4 receptor by MRAP2 proteins in zebrafish. Science 341, 278-281. doi: 10.1126/science.1232995

Soto, A. G., Smith, T. H., Chen, B., Bhattacharya, S., Cordova, I. C., Kenakin, T., et al. (2015). N-linked glycosylation of protease-activated receptor-1 at extracellular loop 2 regulates G-protein signaling bias. Proc. Natl. Acad. Sci. U.S.A. 112, E3600-E3608.

Srisai, D., Yin, T. C., Lee, A. A., Rouault, A. A. J., Pearson, N. A., Grobe, J. L., et al. (2017). MRAP2 regulates ghrelin receptor signaling and hunger sensing. Nat. Commun. 8:713.

van Koppen, C. J., and Nathanson, N. M. (1990). Site-directed mutagenesis of the $\mathrm{m} 2$ muscarinic acetylcholine receptor. Analysis of the role of N-glycosylation in receptor expression and function. J. Biol. Chem. 265, 20887-20892. doi: 10.1016/s0021-9258(17)45299-9

Wang, T., Nakagawa, S., Miyake, T., Setsu, G., Kunisue, S., Goto, K., et al. (2020). Identification and functional characterisation of N-linked glycosylation of the orphan G protein-coupled receptor Gpr176. Sci. Rep. 10:4429.

Yin, T. C., Bauchle, C. J., Rouault, A. A. J., Stephens, S. B., and Sebag, J. A. (2020). The insulinostatic effect of ghrelin requires MRAP2 expression in delta cells. iScience 23:101216. doi: 10.1016/j.isci.2020.101216

Conflict of Interest: The authors declare that the research was conducted in the absence of any commercial or financial relationships that could be construed as a potential conflict of interest.

Publisher's Note: All claims expressed in this article are solely those of the authors and do not necessarily represent those of their affiliated organizations, or those of the publisher, the editors and the reviewers. Any product that may be evaluated in this article, or claim that may be made by its manufacturer, is not guaranteed or endorsed by the publisher.

Copyright (c) 2021 Verdinez and Sebag. This is an open-access article distributed under the terms of the Creative Commons Attribution License (CC BY). The use, distribution or reproduction in other forums is permitted, provided the original author(s) and the copyright owner(s) are credited and that the original publication in this journal is cited, in accordance with accepted academic practice. No use, distribution or reproduction is permitted which does not comply with these terms. 